\title{
A Two-Period Newsvendor Model with Product Extension and Shortage-Making Strategy
}

\author{
Kuo-Hsien Wang, ${ }^{1}$ Che-Tsung Tung, ${ }^{2}$ and Yuan-Chih Huang ${ }^{1}$ \\ ${ }^{1}$ Department of Business Administration, Takming University of Science and Technology, No. 56, Section 1, \\ Huanshan Road, Neihu District, Taipei 11451, Taiwan \\ ${ }^{2}$ Department of International Trade, Takming University of Science and Technology, No. 56, Section 1, \\ Huanshan Road, Neihu District, Taipei 11451, Taiwan
}

Correspondence should be addressed to Che-Tsung Tung; dennistung@takming.edu.tw

Received 25 April 2013; Accepted 17 June 2013

Academic Editors: C. Mohan, L. Tadj, and X.-M. Yuan

Copyright (C) 2013 Kuo-Hsien Wang et al. This is an open access article distributed under the Creative Commons Attribution License, which permits unrestricted use, distribution, and reproduction in any medium, provided the original work is properly cited.

\begin{abstract}
This study deals with a two-period newsvendor setting in which the item in the second period is a product extension of the item in the first period. A shortage strategy toward the first item is intentionally made so as to stimulate more sales amounts of the second item. The stochastic demand of these two items is assumed to be a linear-additive pattern comprising a deterministic demand and an error demand, where the deterministic demand consists of a primary demand and a consumer price elasticity, and the error demand is hypothesized to be exponentially distributed. The objective of this study is to optimize system's overall expected profit by jointly determining the optimal order quantities and selling prices of these two items. We first compare our proposed model with the classical newsvendor model in light of profit performances, and it reveals that a higher shifting demand rate makes our model a more profitable setting. Impact on profit performances caused by an increasing primary demand of the second item is then demonstrated by numerical examples that an unthought-of ripple effect of an increasing error demand of the second item also occurs.
\end{abstract}

\section{Introduction}

With respect to a newsvendor-type item, introducing a series of its homogeneous items one after another is getting an overwhelming predominant edge in the competitive markets. A variety of items, such as a series of smartphones of iPhone, Galaxy, and hTC, are among the illustrations. And obviously, its main purpose is to draw more potential buyers as well as to strive for better market shares. Meanwhile, as we have observed from the markets, a strategy of shortage-making toward a prior item is usually deliberately created to motivate demand on its following homogeneous items. All together extends a classical single-period newsvendor setting to a multiperiod one with implementations of a shortage strategy in prior period, and a product extension in upcoming period is beginning to mushroom in modern marketing approaches.

For any newsvendor item, referring to Arcelus et al. [1], its stochastic demand could be in a linear-additive pattern consisting of a deterministic demand and an error demand, where the deterministic demand is composed of a primary demand and a consumer price elasticity. In practice, amounts of the primary demand of an item could be predicted by virtue of observation, surveys, or experimentation from the markets; and its size, according to practical experiences, mostly depends on its company goodwill, product quality, product applicability, and the degree of customer acceptance. The result is a generation of the concept of "product extension." A product extension is defined as a new introduction of an item with additional or upgraded features or different dimensions in comparison with its prior item. Clearly, taking full advantage of sales information of a prior introduced item to develop and modify its next upgraded and sophisticated product extension definitely could secure more customers and efficiently improve the product extension's primary demand as well, and this spotlights a vital importance of the concept of product extension in a multiperiod newsvendor setting. 
Most existing publications, however, have zeroed in on the classical newsvendor problem, that is, a single-period setting without implementations of a strategy of shortagemaking and a concept of product extension. For example, $\mathrm{H}$. S. Lau and A. H. L. Lau [2] discussed manufacturer's pricing strategy and return policy for a single-period commodity. Chung and Flynn [3] focused on a single-period newsboy problem with reactive production. Bose and Anand [4] addressed returns policies with exogenous price for a singleperiod newsvendor problem. Das and Maiti [5] developed an application of bilevel newsboy problem in two substitute items under capital cost. Shi and Chen [6] investigated Pareto optimal contracts for a supply chain with satisficing objectives under a frame of a single-period setting. Serin [7] explored competitive newsvendor problems with the same Nash and Stackelberg solutions, and Yang et al. [8] studied optimal ordering and pricing decisions for a target-oriented singleperiod newsvendor. An article more related to ours was by Halati and $\mathrm{He}$ [9] who first analyzed a single-period newsvendor problem with quantity-based fixed incentives and then subsequently extended to a multiperiod newsvendor problem without considering shortage strategy and product extension. Plus, You and Chen [10] handled an inventory management problem and selling price decision for perishable products over a finite time planning horizon with an assumption of a time- and price-dependent demand. Recently, Wang and Tung [11], inspired by a behavior of population growth rate, first constructed a time-dependent demand model for gradually obsolescent products that face a problem of short lifespan and then designed a strategy of finite times of discount to promote those obsolescent products when demand begins to fade out.

In this study we tackle a two-period newsvendor setting. The item in the second period is a product extension of the item in the first period. And a shortage strategy in the first period is intentionally implemented for a purpose of demand stimulation of the second item in the second period. Shortage costs due to unsatisfied demands in the two periods and salvage value for the second item's excess inventory are also, respectively, taken into account. The goal of this study is to determine the optimal order quantities and selling prices of these two items that maximize the two-period setting's overall expected profit. Main contributions of that are twofold: (1) unlike the classical single-period newsvendor setting, we pioneer in incorporating the concept of product extension and the strategy of shortage making into an innovative twoperiod newsvendor setting; and (2) compared to the classical newsvendor model, our proposed model produces a remarkable profit performance after integrating the two elements of product extension and shortage strategy into our strategic two-period newsvendor setting.

The rest of this study is organized as follows. Assumptions and notation are given in Section 2 where we first review the pertinent classical newsvendor model and then construct our two-period newsvendor model, along with theoretical analysis; they optimize the expected profit. Numerical examples and managerial insights are accordingly undertaken in Section 3. Finally, remarks and further potential researches are presented to end this study.

\section{The Models}

The scenario of this study is described as follows. Two homogeneous newsvendor-type items, say item 1 and item 2, will be, respectively, sold in two distinct selling periods. The item 2 is a product extension of the item 1 . In order to lure more sales amount for the item 2 in the second period, a shortagemaking strategy toward the item 1 is implemented in the first period. For item $i, i=1,2$, its stochastic demand is assumed to be a linear-additive form of $x_{i}=D_{i}+\varepsilon_{i}$, where $D_{i}=\alpha_{i}-\beta_{i} p_{i}$ is a deterministic demand with a primary demand $\alpha_{i}$, consumer's price-elasticity $\beta_{i}$, and a selling price $p_{i} ; \varepsilon_{i} \in[0, \infty)$ is an error demand assumed to be an exponential distribution with a pdf $f_{i}\left(\varepsilon_{i}\right)$, a cdf $F_{i}\left(\varepsilon_{i}\right)$, and a mean $\mu_{i}$. Unit purchasing cost and unit shortage cost due to unsatisfied demand of the two items are $c_{i}$ and $s_{i}$, respectively; the over stock of item 2 will be saved at a price $b$ per unit at the end of the second period.

2.1. The Classical Newsvendor Model (Classical NV). First, we extend the classical single-period newsvendor model into a two-period newsvendor model in which there is neither the strategy of short making nor the concept of product extension. Thus for item $i, i=1,2$, we define $z_{i}$ as a safety stock level responding to the error demand, and let $Q_{i}=D_{i}+z_{i}$ be its order quantity; its profit then is calculated by

$$
\begin{aligned}
\pi_{i}^{c}= & \left(p_{i}-c_{i}\right)\left(D_{i}+\varepsilon_{i}\right)-c_{i}\left(z_{i}-\varepsilon_{i}\right)^{+} \\
& -\left(p_{i}-c_{i}+s_{i}\right)\left(\varepsilon_{i}-z_{i}\right)^{+}
\end{aligned}
$$

where $\{x\}^{+}=\max \{x, 0\}$, and its expected profit is given by

$$
\begin{aligned}
E\left[\pi_{i}^{c}\right]= & \left(p_{i}-c_{i}\right)\left(D_{i}+\mu_{i}\right)-c_{i} \Lambda_{i}\left(z_{i}\right) \\
& -\left(p_{i}-c_{i}+s_{i}\right) \Theta_{i}\left(z_{i}\right),
\end{aligned}
$$

where $\Lambda_{i}\left(z_{i}\right)=\int_{0}^{z_{i}}\left(z_{i}-\varepsilon_{i}\right) f_{i}\left(\varepsilon_{i}\right) d \varepsilon_{i}, \Theta_{i}\left(z_{i}\right)=\int_{z_{i}}^{\infty}\left(\varepsilon_{i}-\right.$ $\left.z_{i}\right) f_{i}\left(\varepsilon_{i}\right) d \varepsilon_{i}$; thus an overall expected profit for the classical $\mathrm{NV}$ is obtained by

$$
\begin{aligned}
E\left[\pi^{c}\right]= & \sum_{i=1}^{2}\left(p_{i}-c_{i}\right)\left(D_{i}+\mu_{i}\right)-\sum_{i=1}^{2} c_{i} \Lambda_{i}\left(z_{i}\right) \\
& -\sum_{i=1}^{2}\left(p_{i}-c_{i}+s_{i}\right) \Theta_{i}\left(z_{i}\right) .
\end{aligned}
$$

2.2. The Shortage-Making Newsvendor Model (Our NV). Recall that a shortage strategy is projected to be made in the first period; we thus discard all its error demand to ensure occurrence of the shortage. That way let $Q_{1}=D_{1}=\alpha_{1}-\beta_{1} p_{1}$ be the item first order size, yielding an expected profit as follows:

$$
E\left[\pi_{1}^{s}\right]=\left(p_{1}-c_{1}\right) D_{1}-s_{1} \mu_{1} .
$$

Equation (4) apparently implies that $\mu_{1}$ is a possible amount of sale losses due to the shortage strategy; therefore, we reasonably assume that $r \mu_{1}$ with a demand shifting rate $r, 0 \leq r \leq 1$, is an additional demand that will shift from 
TABLE 1: Comparison between our NV and the classical NV as $r=0.6-1.0$.

\begin{tabular}{lcccccccc}
\hline & & $p_{1}$ & $p_{2}$ & $Q_{1}$ & $Q_{2}$ & $E_{1}$ & $E_{2}$ \\
\hline Classical NV & & 58.8 & 58.8 & 265.7 & 265.7 & 10485.2 & 10485.2 & 20970.4 \\
& $r=0.6$ & 56.0 & 60.9 & 220.0 & 284.3 & 9246.7 & 11548.7 & 20795.4 \\
Our NV & $r=0.7$ & 56.0 & 61.2 & 220.0 & 286.1 & 9246.7 & 11712.3 & 20959.0 \\
& $r=0.8$ & 56.0 & 61.6 & 220.0 & 287.9 & 9246.7 & 11877.0 & 21123.7 \\
& $r=0.9$ & 56.0 & 61.9 & 220.0 & 289.7 & 9246.7 & 12042.9 & 21289.5 \\
& $r=1.0$ & 56.0 & 62.3 & 220.0 & 291.5 & 9246.7 & 12209.8 & 21456.5 \\
\hline
\end{tabular}

the first period to the second period, totaling the item second demand up to $x_{2}+r \mu_{1}=D_{2}+r \mu_{1}+\varepsilon_{2}$. Likewise, let $Q_{2}=$ $D_{2}+r \mu_{1}+z_{2}$ be its order size; profit is then computed by

$$
\begin{aligned}
\pi_{2}^{s}= & \left(p_{2}-c_{2}\right)\left(D_{2}+r \mu_{1}+\varepsilon_{2}\right)-\left(c_{2}-b\right)\left(z_{2}-\varepsilon_{2}\right)^{+} \\
& -\left(p_{2}-c_{2}+s_{2}\right)\left(\varepsilon_{2}-z_{2}\right)^{+} .
\end{aligned}
$$

And its expected profit is by

$$
\begin{aligned}
E\left[\pi_{2}^{s}\right]= & \left(p_{2}-c_{2}\right)\left(D_{2}+r \mu_{1}+\mu_{2}\right)-\left(c_{2}-b\right) \Lambda_{2}\left(z_{2}\right) \\
& -\left(p_{2}-c_{2}+s_{2}\right) \Theta_{2}\left(z_{2}\right) .
\end{aligned}
$$

Finally, adding $E\left[\pi_{1}^{s}\right]$ and $E\left[\pi_{2}^{s}\right]$ yields our NV's overall expected profit by

$$
\begin{aligned}
E\left[\pi^{s}\right]= & \left(p_{1}-c_{1}\right) D_{1}+\left(p_{2}-c_{2}\right)\left(D_{2}+\mu_{2}\right) \\
& -\left(c_{2}-b\right) \Lambda_{2}\left(z_{2}\right)-\left(p_{2}-c_{2}+s_{2}\right) \Theta_{2}\left(z_{2}\right) \\
& +\left(r\left(p_{2}-c_{2}\right)-s_{1}\right) \mu_{1} .
\end{aligned}
$$

2.3. Analysis. Since the expected profit $E\left[\pi^{s}\right]$ is a function of $z_{2}, p_{1}$, and $p_{2}$, we, respectively, take its first- and secondorder partial derivatives as follows:

$$
\begin{gathered}
\frac{\partial E\left[\pi^{s}\right]}{\partial z_{2}}=\left(p_{2}-c_{2}+s_{2}\right)-\left(p_{2}-b+s_{2}\right) F_{2}\left(z_{2}\right), \\
\frac{\partial E\left[\pi^{s}\right]}{\partial p_{1}}=-2 \beta_{1} p_{1}+\alpha_{1}+c_{1} \beta_{1}, \\
\frac{\partial E\left[\pi^{s}\right]}{\partial p_{2}}=-2 \beta_{2} p_{2}+\alpha_{2}+c_{2} \beta_{2}+r \mu_{1}+\mu_{2}-\Theta_{2}\left(z_{2}\right) .
\end{gathered}
$$

Also, $\partial^{2} E\left[\pi^{s}\right] / \partial z_{2}^{2}=-\left(p_{2}-b+s_{2}\right) f_{2}\left(z_{2}\right), \partial^{2} E\left[\pi^{s}\right] / \partial p_{1}^{2}=$ $-2 \beta_{1}, \partial^{2} E\left[\pi^{s}\right] / \partial p_{2}^{2}=-2 \beta_{2}, \partial^{2} E\left[\pi^{s}\right] / \partial z_{2} \partial p_{1}=0, \partial^{2} E\left[\pi^{s}\right] /$ $\partial z_{2} \partial p_{2}=1-F_{2}\left(z_{2}\right)$, and $\partial^{2} E\left[\pi^{s}\right] / \partial p_{1} \partial p_{2}=0$. Therefore, its corresponding Hessian matrix is expressed by

$$
H=\left(\begin{array}{ccc}
-\left(p_{2}-b+s_{2}\right) f_{2}\left(z_{2}\right) & 0 & 1-F_{2}\left(z_{2}\right) \\
0 & -2 \beta_{1} & 0 \\
1-F_{2}\left(z_{2}\right) & 0 & -2 \beta_{2}
\end{array}\right) .
$$

Furthermore, its first principal minor is $\left|H_{11}\right|=-\left(p_{2}-b+\right.$ $\left.s_{2}\right) f_{2}\left(z_{2}\right)<0$; its second principal minor is $\left|H_{22}\right|=2 \beta_{1}\left(p_{2}-\right.$ $\left.b+s_{2}\right) f_{2}\left(z_{2}\right)>0$; its third principal minor is by $\left|H_{33}\right|=$ $-2 \beta_{1}\left(2 \beta_{2}\left(p_{2}-b+s_{2}\right) f_{2}\left(z_{2}\right)-\left(1-F_{2}\left(z_{2}\right)\right)^{2}\right)<0$ if $\beta_{2}\left(p_{2}-b+\right.$ $\left.s_{2}\right) f_{2}\left(z_{2}\right)>1 / 2$ holds. We accordingly come to a conclusion that our NV's expected profit $E\left[\pi^{s}\right]$ is jointly concave in $z_{2}, p_{1}$, and $p_{2}$ as long as $\beta_{2}\left(p_{2}-b+s_{2}\right) f_{2}\left(z_{2}\right)>1 / 2$, which enables us to proceed with numerical examples in next section.

\section{Examples}

We first clarify that, for item $i, i=1,2$, its error demand is assumed to be an exponential distribution with a pdf $f_{i}\left(\varepsilon_{i}\right)=$ $\lambda_{i} e^{-\lambda_{i} \varepsilon_{i}}$, and according to optimal first-order necessary conditions, the optimal values $z_{1}, z_{2}, p_{1}$, and $p_{2}$ for the classical NV and the optimal values $z_{2}, p_{1}$, and $p_{2}$ for our NV could be obtained by, respectively, solving the following systems of equations.

Optimal values for the classical NV satisfy

$$
\begin{aligned}
& -2 \beta_{i} p_{i}+\alpha_{i}+c_{i} \beta_{i}+\frac{1}{\lambda_{i}}\left(1-e^{-\lambda_{i} z_{i}}\right)=0, \\
& \left(p_{i}-c_{i}+s_{i}\right)-\left(p_{i}+s_{i}\right)\left(1-e^{-\lambda_{i} z_{i}}\right)=0 .
\end{aligned}
$$

Optimal values for our NV satisfy

$$
\begin{aligned}
& \left(p_{2}-b+s_{2}\right) e^{-\lambda_{2} z_{2}}-\left(c_{2}-b\right)=0, \\
& p_{1}=\frac{1}{2 \beta_{1}}\left(\alpha_{1}+c_{1} \beta_{1}\right), \\
& p_{2}=\frac{1}{2 \beta_{2}}\left(\alpha_{2}+c_{2} \beta_{2}+\frac{r}{\lambda_{1}}+\frac{1-e^{-\lambda_{2} z_{2}}}{\lambda_{2}}\right) .
\end{aligned}
$$

In order to illustrate how our NV will impact on profit performances, the same parameter values for both items are hypothesized, which includes $\alpha_{1}=\alpha_{2}=500, \beta_{1}=\beta_{2}=5$, $c_{1}=c_{2}=12, s_{1}=s_{2}=13, \lambda_{1}=\lambda_{2}=0.03$, and $b=3$. For simplicity, respectively, let $E_{1}$ and $E_{2}$ be the expected profits of the two periods, and $E=E_{1}+E_{2}$ is the overall expected profit for the classical two-period NV and our two-period newsvendor NV. Noteworthily, the values of $s_{i}>c_{i}, i=1,2$ are on purpose set to genuinely reflect the influence of our shortage-making strategy on our NV.

Example 1. This example (see Table 1) makes a comparison between our NV and the classical NV in respect of the optimal selling prices, optimal order sizes, and optimal expected profits as the shifting demand rate $r=0.6-1.0$.

Not surprisingly, compared to the classical NV, our NV indicates a lower $p_{1}$, a less $Q_{1}$, and a worse $E_{1}$ in the first period as a result of discard of the first item's error demand. More importantly, Table 1 also identifies a consistent result with our expectation that a higher shifting demand rate $r$ allows our NV to set a higher selling price $p_{2}$, to place a higher order quantity $Q_{2}$, and to generate a better expected profit $E_{2}$ in the second period and eventually a brilliant overall expected profit $E$ in our strategic two-period setting as well. Nevertheless, Table 1 unveils that our NV's expected profits 
TABLE 2: Impact of $\alpha_{2}$ increased by $2 \%$ at a time; $r=0.8$.

\begin{tabular}{ccccccccc}
\hline & $p_{1}$ & $p_{2}$ & $z_{2}$ & $Q_{1}$ & $Q_{2}$ & $E_{1}$ & $E_{2}$ \\
\hline \multirow{5}{*}{$\alpha_{2}=500$} & 56.0 & 61.6 & 69.1 & 220.0 & 287.9 & 9246.7 & 11877.0 & 21123.7 \\
& 56.0 & 62.6 & 69.6 & 220.0 & 293.3 & 9246.7 & 12377.9 & 21624.5 \\
& 56.0 & 63.5 & 70.0 & 220.0 & 298.7 & 9246.7 & 12888.8 & 22135.4 \\
& 56.0 & 64.6 & 70.5 & 220.0 & 304.2 & 9246.7 & 13409.7 & 22656.4 \\
& 56.0 & 65.6 & 70.9 & 220.0 & 309.6 & 9246.7 & 13940.7 & 23187.4 \\
& 56.0 & 66.4 & 71.4 & 220.0 & 315.0 & 9246.7 & 14481.8 & 23728.5 \\
\hline
\end{tabular}

will overshadow the classical NV's only when $r \geq 0.8$, equivalently implicating that our shortage-making strategy can only apply to items that possess higher shifting demand rate $r$.

Example 2. This example (see Table 2) is in response to how an increasing primary demand of the second item will affect our NV's profit performances, thus we enlarge the item second primary demand $\alpha_{2}$ by $2 \%$ at a time and remain the other parameter values unchanged.

Table 2 first verifies an outcome in line with our anticipation that a larger primary demand urges a policymaker to set a higher selling price, which results in a better overall expected profit $E$. Also, a noticeable finding is that a $10 \%$ increase in the primary demand $\alpha_{2}$ will simultaneously trigger a ripple effect of an increasing amount $z_{2}$ in the error demand from 69.1 to 71.4 as shown in Table 2 , which is quite conceivable as a large primary demand literally stands for its high product quality, various applicability, and high degree of customer acceptance; all of which will help stimulate and facilitate much more unexpected, potential, and new customers with great possibilities. On the whole, not only can our NV prompt a higher selling, but also a more error demand in the second period as long as the effect of an increasing primary demand of the second item occurs as a result of the shortage of the first item. And this explains why Table 2 displays a $12.3 \%$ increase in our NV's expected profit $E$ from 21123.7 to 23728.5 , whereas the primary demand $\alpha_{2}$ only increases $10 \%$.

\section{Conclusion}

Other than the classical single-period newsvendor setting, we investigated a two-period newsvendor setting in connection with a product extension and a shortage-making strategy; namely, that item in the second period is a product extension of that item in the first period where a shortage is intentionally made for the purpose of boosting more sales amounts in the second period. The advantages of our NV include the following: (1) sales information acquired from the first item, such as market response and sales status, would provide a significant revision guideline to modify and upgrade its homogeneous product in the second period, and (2) an increasing primary demand, a higher selling price, and an increasing error demand in the second period could likely be elicited due to shortage strategy in the first period. The consequence is the contribution of an outstanding profit performance to our strategic NV versus the classical NV. Nonetheless, experiments have shown that only items with high shifting demand rate can apply to our shortage-making strategy.
Still, two practical and interesting directions linked with our proposed newsvendor problem are worthy of further exploring. The first is to extend our two-period setting to a multiperiod setting, and how to use shortage strategies in this multiple selling periods system and how to ensure the occurrence of each shortage in each individual selling period are our main concerns. The second is to modify our strategic newsvendor model to a supply chain model with a returnpolicy commitment offered by upstream manufacturers, which could be in one-manufacturer-and-one-retailer or one-manufacturer-and-multiretailer type of newsvendor setting.

\section{References}

[1] F. J. Arcelus, S. Kumar, and G. Srinivasan, "Evaluating manufacturer's buyback policies in a single-period two-echelon framework under price-dependent stochastic demand," Omega, vol. 36, no. 5, pp. 808-824, 2008.

[2] H. S. Lau and A. H. L. Lau, "Manufacturer's pricing strategy and return policy for a single-period commodity," European Journal of Operational Research, vol. 116, no. 2, pp. 291-304, 1999.

[3] C. S. Chung and J. Flynn, "A newsboy problem with reactive production," Computers and Operations Research, vol. 28, no. 8, pp. 751-765, 2001.

[4] I. Bose and P. Anand, "On returns policies with exogenous price," European Journal of Operational Research, vol. 178, no. 3, pp. 782-788, 2007.

[5] B. Das and M. Maiti, "An application of bi-level newsboy problem in two substitutable items under capital cost," Applied Mathematics and Computation, vol. 190, no. 1, pp. 410-422, 2007.

[6] C. Shi and B. Chen, "Pareto-optimal contracts for a supply chain with satisficing objectives," Journal of the Operational Research Society, vol. 58, no. 6, pp. 751-759, 2007.

[7] Y. Serin, "Competitive newsvendor problems with the same Nash and Stackelberg solutions," Operations Research Letters, vol. 35, no. 1, pp. 83-94, 2007.

[8] S. Yang, C. V. Shi, and X. Zhao, "Optimal ordering and pricing decisions for a target oriented newsvendor," Omega, vol. 39, no. 1, pp. 110-115, 2011.

[9] A. Halati and Y. He, "Analysis of supply chains with quantity based fixed incentives," European Journal of Operational Research, vol. 202, no. 1, pp. 214-222, 2010.

[10] P. S. You and T. C. Chen, "Dynamic pricing of seasonal goods with spot and forward purchase demands," Computers and Mathematics with Applications, vol. 54, no. 4, pp. 490-498, 2007.

[11] K. H. Wang and C. T. Tung, "Construction of a model towards EOQ and pricing strategy for gradually obsolescent products," Applied Mathematics and Computation, vol. 217, no. 16, pp. 6926-6933, 2011. 


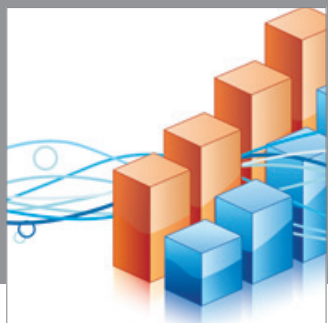

Advances in

Operations Research

mansans

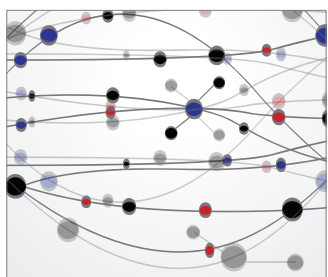

The Scientific World Journal
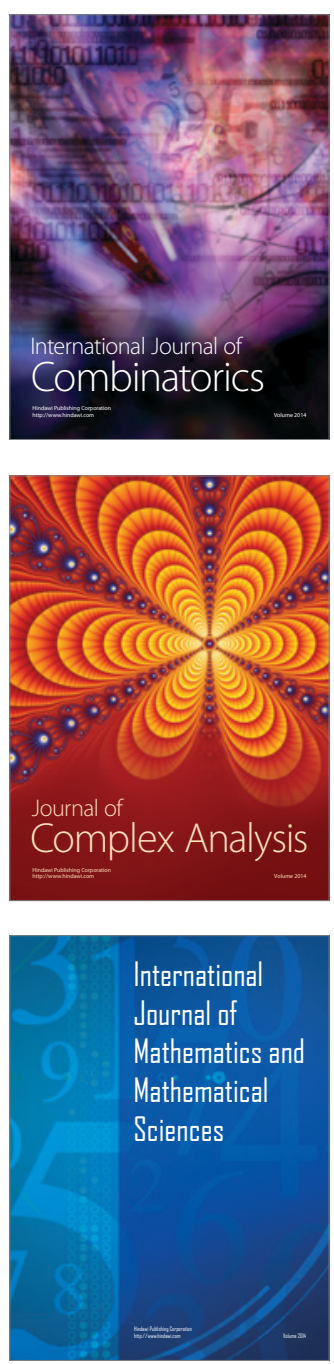
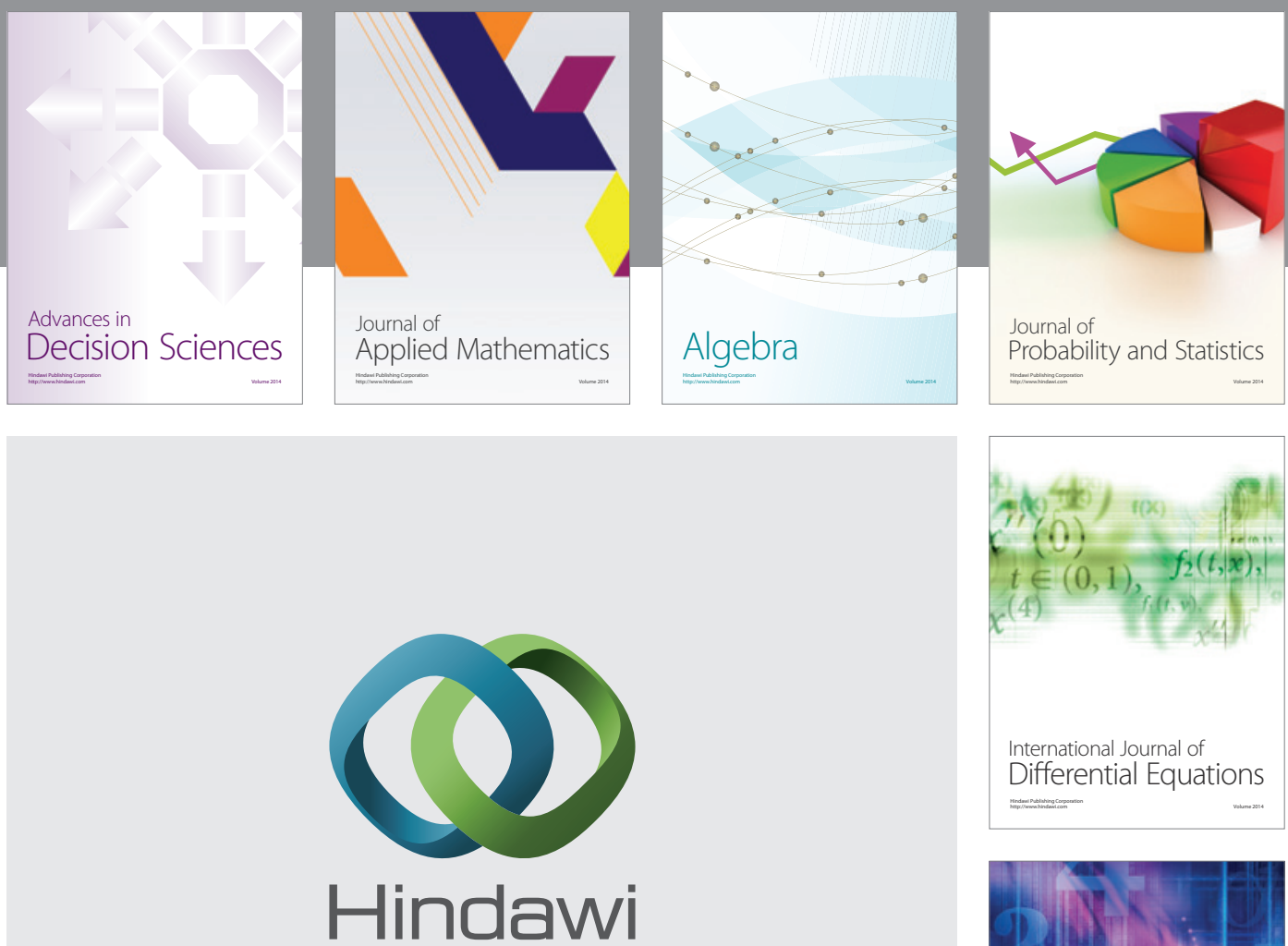

Submit your manuscripts at http://www.hindawi.com
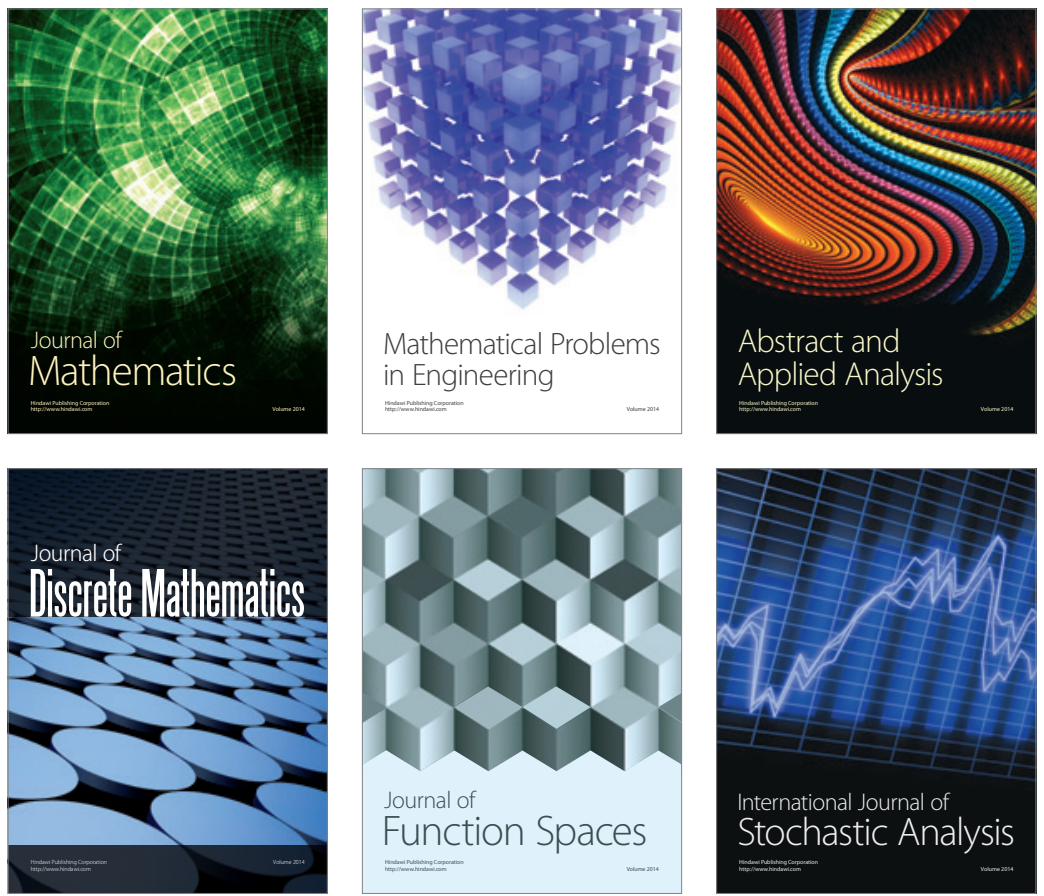

Journal of

Function Spaces

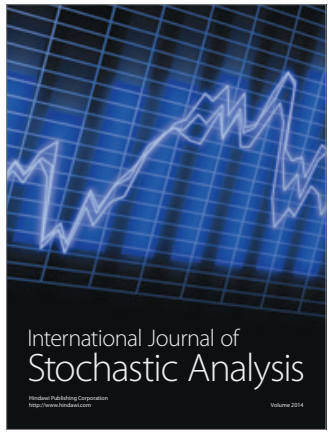

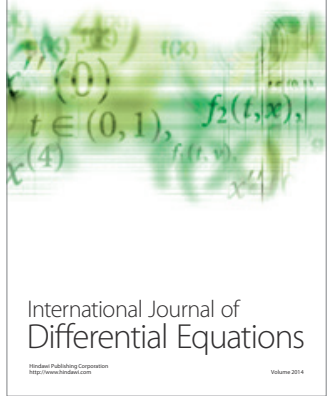
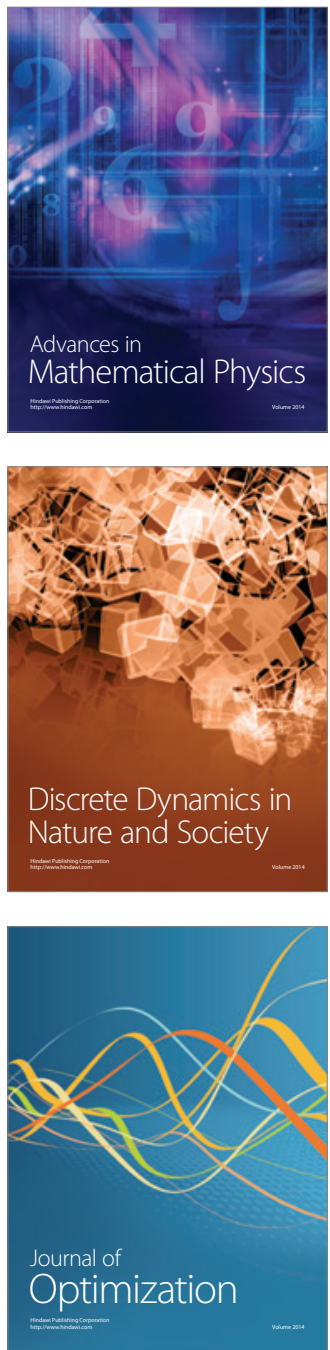